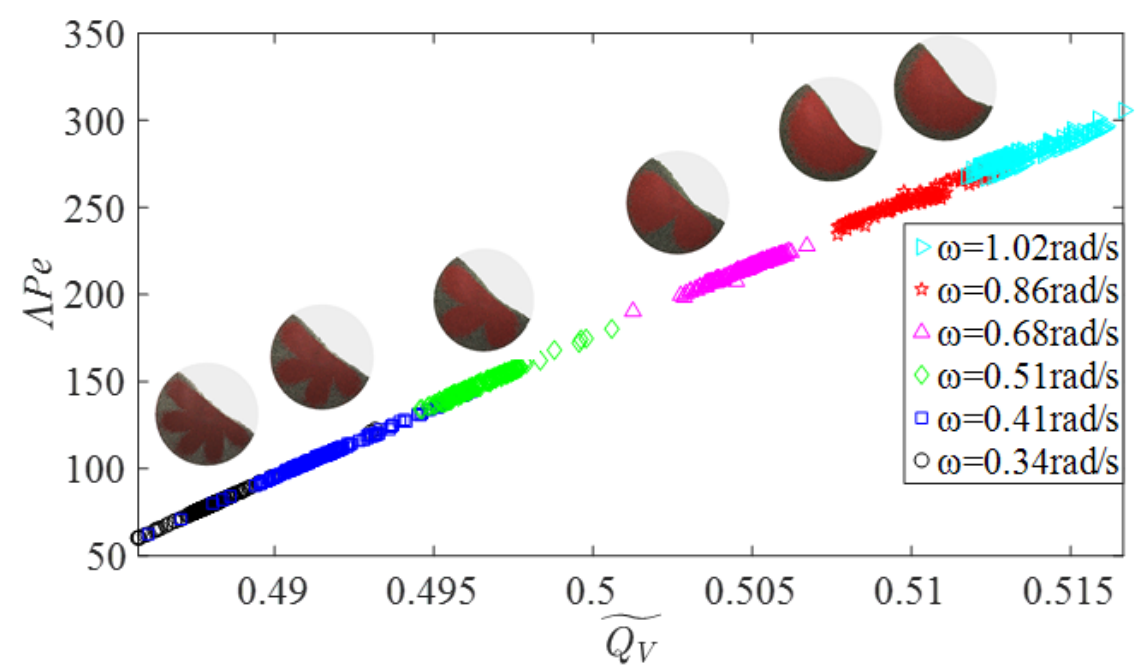

$\Lambda P e$ vs $\widetilde{Q_{V}}$ under different segregation patterns with respect to different rotational speeds. 


\begin{abstract}
There is a paucity of studies on the relationship between the control parameters and kinetic parameters in binary-size granular segregation models. In this study, the machine vision method and particle image velocimetry (PIV) were used to measure the shape index of segregation pattern and streamwise velocity, respectively. Two scaled parameters, control parameters and kinetic parameters, were calculated based on the continuous flow model and segregation model. The control parameters include volume flow rate, and the kinetic parameters include the ratio of segregation effects to diffusion effects. By analyzing the relationship between the two parameters under the core and streak patterns, the configuration of segregation pattern at steadystate changes with the control parameters wherein the ratio of segregation effects to diffusion effects decreases approximately linearly as the volume flow rate decreases. These experimental results aid in revealing the mechanism of segregation and providing the reference data for improving the theoretical models.
\end{abstract}




\title{
Binary-size granules segregation from core pattern to streak pattern in a rotating drum
}

\author{
W. Z. Xiu a , R. Li ${ }^{\text {ba }}$, Q. Chen ${ }^{\mathrm{a}}$, Q. C.Sun ${ }^{\mathrm{c}}$, V. Zivkovic ${ }^{\mathrm{d}}$, and H. Yang ${ }^{\mathrm{a} *}$

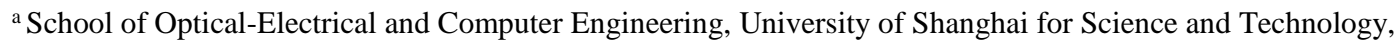 \\ Shanghai, 200093, China \\ ${ }^{\mathrm{b}}$ School of Medical instrument and food engineering, University of Shanghai for Science and Technology, \\ Shanghai, 200093, China \\ ${ }^{\mathrm{c}}$ State Key Laboratory of Hydroscience and Engineering, Tsinghua University, Beijing 100084, China \\ ${ }^{\mathrm{d}}$ School of Engineering, Newcastle University, NE1 7RU, United Kingdom \\ Corresponding author: yanghui@ usst.edu.cn (+86-021-55274344)
}

\section{Introduction}

Segregation is a commonly observed during granular mixing of multi-sized particles and causes various patterns, such as the debris and gravelly bed formation in nature. Granular segregation occurs in numerous industrial, pharmaceutical, and food processes, which often leads to undesirable effects on the quality of products $[1,2]$. It is easy to measure and control a granular flow experiment in a quasi-two-dimensional rotating drum, which is a main method for examining granular segregation [2]. When granules are mixed, segregation pattern is often formed due to granular flow [3,35]. With respect to different segregation patterns [26], the relationship between the control parameter (system size, granular size, and rotating speed) and kinetic parameter (diffusion 
coefficient, flow layer thickness, and viscosity) is not characterized. Therefore, the establishment of a rheological relationship requires the realization of an accordant understanding of this relationship $[4,5-12]$

In recent years, several researchers have examined granular segregation and mixing in various systems. In 2000, Ottino et al. [13] presented segregation mechanisms and models for size and density segregation and introduced a chaotic advection framework for examining granular mixing/segregation in circular and non-circular rotating drums. Currently, the granular segregation model is classified into two types, namely the kinetic sieving model and thermal diffusion model. The principles of the kinetic sieving model are widely accepted for size separation under the effect of gravity $[14,15]$. In the granular system, under shear flow, the particles are staggered with each other at different flow depths, thereby causing continuous as well as random changes in the internal force chain network and particle spacing. This leads to the formation of a series of interparticle gaps, which increases the probability of large and small granules falling in the gap owing to gravity. However, small granules are more likely to fall in the formed gaps under gravity, whereas large granules rise to the flow surface. Kwok et al. confirmed this asymmetric motion micromechanics for coarse and fine granules using the discrete element method (DEM) simulation and theoretical analysis [16].

The thermal diffusion model is based on the granular kinetic theory. Jenkins et al. applied the kinetic theory of granular flow on granular mixtures containing different granule sizes or masses [17] and derived the effect of collision between two types of particles of different sizes or masses (binary mixtures) on granular pressure and flux. Based on the kinetic theory, segregation flux is 
expressed as the diffusion equation related to granular temperature. Hence, these types of models were classified as thermal diffusion models. Khakhar et al. [18] examined the kinetic theory during the mixing process using tracer particles and image analysis methods in a quasi-two-dimensional rotating drum. They observed that the mixing rate increased with the decrease in the filling level as a control parameter. In 2000, McCarthy et al. $[19,20]$ simulated segregation patterns in rotating drums of different shapes (control parameter) and observed that the perimeter length of the patterns grew exponentially with revolutions in ellipse- and square-shaped drums, whereas the perimeter grew linearly with revolutions in the common circular drum. In 2005, Jain et al. [21] examined granular segregation in a quasi-two-dimensional rotating drum at a typical filling rate of $50 \%$ and analyzed the effects of particle size ratio (control parameter) of steel and glass beads on segregation. They hypothesized that percolation leads to the formation of a small core of small beads. Moreover, an increase in size ratio leads to the domination of percolation over mixing. Thus, the core of small beads became larger, whereas the streaks of small beads at the periphery disappeared. However, granular mixing occurred only when the heavy particles are substantially larger than the light particles.

Hill et al. [22] examined granular segregation using the Poincare section and image method in a quasi-two-dimensional rotating drum and indicated that the effect of diffusion along the depth can be described using the Peclet number $(P e)$. They correlated the intensity of granular segregation and the configuration of the segregation pattern with the segregation index and the shape index respectively. Their calculation model was based on density segregation and did not consider the effects of system size and granule size (control parameters) on segregation. Schlick et al. [23] quantitatively analyzed size segregation using DEM and image processing method for validation 
experiments in a quasi-2D bounded heap. They used the advection-diffusion transport equation to describe segregation in a flow layer to obtain an approximate relationship between the control parameters (system size and particle size) and the kinetic parameters (flow layer depth, diffusion coefficient, and percolation length scale). The proposed relationship can be used to determine the values of control parameters that can result in a mixed or segregated heap. Schlick et al. [24] simulated bidisperse size segregation of a granular material in a quasi-two-dimensional rotating drum using DEM and advection-diffusion transport equation. They used an additional term in the advection-diffusion transport equation to account for segregation for predicting the segregation patterns in a steady state. The results indicated that the degree of segregation increases with the ratio of segregation effects to diffusion effects, as quantified by the non-dimensional group $(\Lambda P e)$. Therefore, there is a need to further study the relationship between the kinetic parameters and the control parameters for understanding granular segregation in a rotating drum as a simple model of granular flow to validate theoretical models.

In this study, we quantified the relationship between scaled volume flow rate (as a control parameter) and ratio of segregation effects to diffusion effects (as a kinetic parameter). Additionally, we explained the mechanism of segregation pattern formation. The experimental set-up and methods are presented in Section 2, whereas Section 3 presents the detailed results and discussion. Finally, the conclusion is presented in Section 4.

\section{Theoretical model of segregation}

Figure 1 is a schematic representation of a drum with radius $R$ rotating clockwise at an angular velocity $\omega$. Granular flow in the rotating drum can be divided into two regions, namely flow layer 
(above the dotted line) and the so-called passive region with almost solid-body dynamics (below the dotted line). The $x$-coordinate represents the streamwise direction, whereas the $y$-coordinate is normal to the flow surface, as shown in the schematic. The origin of the coordinate system is set at the middle of the flow surface, and $u$ and $w$ are the velocities along the $x$ and $y$ directions, respectively. $L$ denotes the length of the drum from the center $(x=0)$ of the flow surface to the wall of the rotating drum for the initial filling state and $h$ denotes the distance from the origin to the axis of rotation. When the rotating speed is high, the granules flow in the flow layer at a steady volume flow rate and form a core pattern. When the rotating speed is low, large and small granules flow through the flow layer and the slope of the boundary between large and small granules in the flow layer alternates between a positive and negative value. This causes wave breaking $[25,26]$, which creates a streak pattern.

To establish the segregation model in a quasi-two-dimensional rotating drum, the advectiondiffusion transport equation [24] was applied to the flow layer, as shown in Eq. (1).

$$
\frac{\partial c_{i}}{\partial \tilde{t}}+\tilde{u} \frac{\partial c_{i}}{\partial \tilde{x}}+\widetilde{w} \frac{\partial c_{i}}{\partial \tilde{y}} \pm \Lambda \epsilon \frac{\partial}{\partial \tilde{y}}\left[c_{i}\left(1-c_{i}\right)\right]=\frac{\epsilon^{2}}{P \mathrm{e}} \frac{\partial^{2} c_{i}}{\partial \tilde{y}^{2}}
$$

The concentration of species $i$ is given by $c_{i}$, where the index $i$ refers to small $(i=s)$ or large particles $(i=l)$. Scaled flow layer thickness is defined as $\epsilon=\frac{\delta_{0}}{R}$, where $\delta_{0}$ denotes the maximum thickness of the flow layer at the radius of the drum, i.e., $\delta_{0}=\delta(0)$. The scaled average velocity in the streamwise direction is defined as $\tilde{u}=\bar{u} / \omega R$, where $\bar{u}$ denotes the average streamwise velocity. However, the scaled average velocity along the percolation direction is given by $\widetilde{w}=\bar{w} / \omega R$, where $\bar{w}$ denotes the average percolation velocity. Similarly, $\tilde{x}=x / R$ and $\tilde{y}=y / R$ are the normalized distance along $x$ and $y$ directions, respectively, and $\tilde{t}=t / \omega$ is the scaled time. 
Segregation depends on two scaled kinetic parameters, i.e., the ratio of segregation to advection, $\Lambda=\mathrm{S}\left(1-\epsilon^{2}\right) / \mathrm{R} \epsilon^{3}$, and the ratio of advection to diffusion, $P e=\omega \mathrm{R}^{2} \epsilon^{2} / D_{\mathrm{dif}}$. However, segregation is controlled by $\Lambda$ and diffusion is controlled by $P e$. Therefore, when only local segregation is considered, the scale coefficient of particle size $S$ can be obtained via simulation or experiment $[23,24]$.

$$
S\left(d_{s}, d_{L}\right)=0.26 d_{s} \log \left(\frac{d_{s}}{d_{l}}\right)
$$

The diffusion coefficient $D_{\text {dif }}$ is defined as follows:

$$
D_{\mathrm{dif}}=0.025\left(\frac{2 d_{l} d_{s}}{d_{l}+d_{s}}\right)^{2} \omega \mathrm{L}^{2} / \delta^{2}
$$

where $d_{S}$ and $d_{L}$ denote the size of large and small particles, respectively. Schlick [24] introduced a dimensionless kinetic parameter, the ratio of segregation effects to diffusion effects $(\Lambda P e)$, as a suitable parameter to quantify the influence of diffusion on granular segregation, as shown in Eq. (4).

$$
\Lambda P e=S \omega \mathrm{R}\left(1-\epsilon^{2}\right) / \epsilon D_{\mathrm{dif}}
$$

However, to calculate $A P e, \epsilon$ should be obtained. $\epsilon$ is associated with mass flow rate based on the mass conservation equation [27] as follows:

$$
Q(x)=\rho \bar{u}(x) \delta(x)=\rho_{b} \int_{-\mathrm{L}}^{x}\left[-\omega(\mathrm{h}-\delta(x)) \frac{\partial \delta(x)}{\partial x}-\omega x\right] d x
$$

where $Q(x), \rho, \rho_{b}, \delta$, and $\bar{u}$ denote the mass flow rate, bulk density of particles in flow layer, bulk density of particles in passive region, the thickness of flow layer, and averaged streamwise velocity [28], respectively, However, 


$$
\bar{u}(x)=\frac{1}{\delta(x)} \int_{-\delta(x)}^{0} u(x, y) d y
$$

where $u(x, y)$ denotes streamwise velocity, which can be measured using particle image velocimetry (PIV). By assuming that the bulk density of particles in the flow layer is equal to that in the passive region, the volume flow rate can be obtained using Eq. (6) as follows:

$$
Q_{V}(x)=\bar{u}(x) \delta(x)=\frac{\omega}{2}\left[\mathrm{~h}^{2}-(\mathrm{h}-\delta(x))^{2}\right]+\frac{\omega}{2}\left(\mathrm{~L}^{2}-x^{2}\right)
$$

By calculating the averaged streamwise velocity $\bar{u}(0)$ at the middle of the surface $(x=0)$ and obtaining $\delta(0), Q_{V}(0)$ from Eq. (7), $\Lambda P e$ was calculated using Eq. (4). Dynamic parameter acquisition is difficult and usually requires multiple simulations or experimental tests. Therefore, the relationship between dynamic parameters and control parameters should be quantified. By solving Eq. (4) and Eq. (7) simultaneously, we obtain

$$
\Lambda P e=\frac{2 S \mathrm{R}\left(1-\epsilon^{2}\right)}{\epsilon D\left(\mathrm{~h}^{2}-(\mathrm{h}-\delta(x))^{2}+\mathrm{L}^{2}-x^{2}\right)} Q_{V}(x)
$$

The relationship between $\Lambda P e$ and $Q_{V}$ under different segregation patterns can be reflected in a better manner by scaling the volume flow rate, i.e., $\widetilde{Q_{V}}=Q_{V} / \omega \mathrm{R}^{2}$. Furthermore, the measuring position is located at the middle of the flow layer position $(x=0)$. Thus, Eq. (8) can be expressed as follows:

$$
\Lambda P e=\frac{2 S\left(1-\epsilon^{2}\right) \omega \mathrm{R}^{3}}{\epsilon D_{\mathrm{dif}}\left(\mathrm{h}^{2}-(\mathrm{h}-\epsilon \mathrm{R})^{2}+\mathrm{L}^{2}\right)} \widetilde{Q_{V}} .
$$

To quantify the relationships between $\Lambda P e$ and $\widetilde{Q_{V}}$ under different segregation patterns, we performed a binary-size granular segregation experiment in a quasi-two-dimensional rotating drum. 


\section{Experiment}

The experimental set-up consisted of a quasi-two-dimensional rotating drum wherein the rotation speed was controlled by a stepping motor (Time Brilliant 86EBP111ALC-TKO). An imaging system with a CCD camera (Revealer 3F04M) and LED (SL-200W) illumination, as shown in Figure 2, was used. The diameter and thickness of the drum were $290 \mathrm{~mm}$ and $10 \mathrm{~mm}$, respectively. Furthermore, the front material of the drum was fabricated from a clear acrylic glass plate with high optical transparency to enable observation and imaging. During the experiment, the imaging exposure time was $200 \mathrm{~ms}$ and the image resolution was $2036 \times 1960$ pixels. The large and small granules were mixed in a volume fraction ratio of 50:50 for the experiment. The large granules were green glass beads having a diameter of $2 \pm 0.1 \mathrm{~mm}$, whereas the small granules were red beads having a diameter of $0.4 \pm 0.02 \mathrm{~mm}$. The density of all the beads was $2400 \mathrm{~kg} / \mathrm{m}^{3}$. Before each experiment, the large and small granules were initially filled side-by-side, as shown in the schematic to a fill level corresponding to $64 \%$.

The experiment was performed by rotating the drum at a high rotating speed (1.02 rad/s) to obtain a core segregation pattern, as shown in Figure 3 (a). The speed of the rotation was then reduced to a desired set value (i.e., $0.86 \mathrm{rad} / \mathrm{s}, 0.68 \mathrm{rad} / \mathrm{s}, 0.51 \mathrm{rad} / \mathrm{s}, 0.41 \mathrm{rad} / \mathrm{s}$, or $0.34 \mathrm{rad} / \mathrm{s}$ ). In this study, the structure segregation pattern was qualified with the shape index $[29,30,31]$ to assess whether the structure of segregation achieved a stable state. Furthermore, the shape index $P^{2} / A$ was measured using the machine vision method, where $P$ is the perimeter of the pattern, and $A$ is the area of the pattern. The shape index can reflect some differences in the segregation pattern structure. When the structure of the patterns reached a stable state, the shape index fluctuated around a certain value in 
our experiment. The segregation pattern was fully developed, as shown in Figure 3 (b) - (e), and the shape index values of streak and core are as shown in Figure 4. The entire process was recorded using the CCD camera at an interval of $20 \mathrm{~s}$ for approximately $1.5 \mathrm{~h}$.

PIV is a well-established technique [32,33,34], which uses statistical correlation methods to track the displacement of a small group of particles. A common approach involves dividing the entire field-of-view into smaller regions, namely interrogation areas. The displacement information between two consecutive images was extracted by cross-correlating the interrogation areas at two sequential time steps by calculating the correlation using Fourier transform. The collected images were pre-processed at the stable state. The entire field-of-view area was set to $1000 \times 1000$ pixels, as shown in Figure 3 (a). The open source algorithm for the PIVlab [32] was used to filter the noise and identify the central position of all particles. This was used to calculate and identify the velocity field. As the granules in the gap were not recognized, small errors occurred, which could be minimized by averaging the velocity along the deep of the flow layer. However, this caused slight fluctuation in the average velocity field. Nevertheless, large systematic errors were avoided. The streamwise velocity was obtained using PIV. Subsequently, the averaged streamwise velocity $\bar{u}(0)$ was calculated using Eq. (6).

\section{Results and discussion}

\subsection{Dependence of segregation pattern on rotating speed}

The segregation patterns under different rotating speeds are shown in Figure 3, wherein the base pattern is shown in Figure 3 (a), the developed pattern after the rotation speed is modulated as shown 
in Figure 3 (b) - (f), and the area of the blue dotted line represents the measurement field of PIV. As the rotating speed decreased, the configurations of the base pattern changed from core to streak. Figure 4 shows the shape index at different rotating speeds. In Figure 4 (a), the value of shape index increases with time. After $500 \mathrm{~s}$, the shape index value fluctuated around a stable value. When the rotating speed was modulated from $1.02 \mathrm{rad} / \mathrm{s}$ to $0.86 \mathrm{rad} / \mathrm{s}$, the value of the shape index changed slightly. However, when the rotating speed was modulated to $0.51 \mathrm{rad} / \mathrm{s}$, the shape index increased significantly, as shown in Figure 4 (c). We measured the velocity when the shape index fluctuated around a stable value. The results are shown in Figure 5.

\subsection{Averaged streamwise velocity}

To calculate $\widetilde{Q_{V}}$ and $\Lambda P e$, we measured the streamwise velocity at the middle of the flow layer using PIV. The moving average filter was used to smoothen the streamwise velocity. The smoothened results of average velocities as a function of time are shown in Figure 5 (a), the time averaged $\bar{u}$ and the errorbar in Figure 5 (b). In the middle of the flow layer, from the core to streak, the time averaged $\bar{u}$ and the errorbar increased as the rotating speed decreased. Two different types of trends are observed in Figure 5 depending on the rotating speed magnitude. At lower speeds, when $\omega$ was lower than $0.68 \mathrm{rad} / \mathrm{s}$, the average streamwise velocity fluctuated significantly as large and small granules alternatively entered the flow layer and formed streak patterns. Specifically, the average streamwise velocity increased when the small beads gradually entered the flow layer. After reaching a certain maximum value, the average streamwise velocity decreased when the large beads gradually entered the flow layer. Thus, small beads exhibited higher fluidity in the flow layer [21]. Conversely, for high rotational speeds, when $\omega$ exceeds $0.86 \mathrm{rad} / \mathrm{s}$, it formed core pattern, large and 
small beads uniformly flowed through the flow layer, and the average streamwise velocity was stable with respect to time. The maximum thickness of the flow layer at the radius of the drum under different rotation speed are shown in Figure 5(c), and the time averaged $\delta_{0}$ are shown in Figure 5(d). As shown in Figure 5(d), in the middle of the flow layer, from the core to streak, the time averaged $\delta_{0}$ decreased as the rotating speed decreased. When the rotational speed is high, $\delta_{0}$ changes steadily with time, forming a core pattern. Gradually decreasing the rotational speed, the value of $\delta_{0}$ will decrease, and fluctuate more violently (errorbar is large), which will result in the change of the configuration of the segregation pattern and the appearance of streaks.

\subsection{Scaled volume flow rate}

By using the streamwise velocity of mixed particles measured using PIV, as shown in Figure 5, the scaled volume flow rate $\widetilde{Q_{V}}$ can be calculated using Eq. (7). Figure 6 shows the average volume flow rate for different rotation speeds. Furthermore, $\widetilde{Q_{V}}$ was averaged in time and indicated using $\left\langle\widetilde{Q_{V}}>\right.$. No matter in high or low rotating speeds, the fluctuation value of $<\widetilde{Q_{V}}>$ is minimal with time. Additionally, there is a significant variation in the streamwise temporal profile shown in Figure 5. However, different patterns exhibited different $\left\langle\widetilde{Q_{V}}>\right.$, which in turn increased with the rotation speed. When the $<\widetilde{Q_{V}}>$ value was lower than 0.508 , the streak pattern was formed. However, as the $<\widetilde{Q_{V}}>$ value decreased, the number of streaks increased, and when the $<\widetilde{Q_{V}}>$ value exceeded 0.508 , the core pattern was formed. The averaged velocity value decreased as the rotating speed increased. However, the scaled depth of flow layer increased with the rotating speed. Thus, the $<\widetilde{Q_{V}}>$ value increased with the rotating speed. 


\subsection{Ratio of segregation effects to diffusion effects}

The dimensionless kinetic parameter $\Lambda P e$ can be calculated using the segregation model expressed in Eq. 9. Figure 7 (a) shows the averaged diffusion coefficient $D_{\text {dif }}$ in time $\left\langle D_{\text {dif }}\right\rangle$ for different rotation speeds. As shown in the figure, the $\left\langle D_{\text {dif }}\right\rangle$ value decreased as $\omega$ increased. However, the averaged diffusion coefficient of the streak pattern was smaller than that of the core pattern. When $\omega$ was lower than $0.51 \mathrm{rad} / \mathrm{s}$, the deviation of averaged diffusion coefficient was large.

Yi Fan used $\Lambda P e$ to describe the effect of the interaction between segregation and diffusion on the pattern structure [23]. Figure 7(b) shows the averaged the ratio of segregation effects to diffusion effects $\langle\Lambda \mathrm{Pe}\rangle$ for different rotation speeds. As the rotation speed decreased from a high value to a lower value, the value of $\langle\Lambda P e\rangle$ decreased, and the deviation became smaller. When the $\langle A P e\rangle$ value is lower than 180 , segregation dominates in the flow layer, and the granular mixture in the drum is segregated in the steady state to form the core pattern. When the $\langle A P e\rangle$ value is between 180 and 300 , the granular mixture in the drum is well mixed in the steady state because diffusion dominates the flow layer and forms the streak pattern.

\subsection{Relationship between $\Lambda P e$ and $\widetilde{Q_{V}}$}

The structure of the patterns formed could be modified by changing the rotation speed. The changes in pattern configuration are closely associated with the control parameters $\left(\widetilde{Q_{V}}\right)$ of the continuous flow model and kinetic parameters $(\Lambda P e)$ of the segregation model. The relationship between $\Lambda P e$ and $\widetilde{Q_{V}}$ under different rotation speeds is shown in Figure 8. The segregation patterns change from the core pattern to streak pattern as $\Lambda P e$ decreases with $\widetilde{Q_{V}}$. By linearly 
fitting the experimental data, $\widetilde{Q_{V}}$ can be expressed as a function of $\Lambda P e$ as follows: $\Lambda P e=$ $7.854 \times 10^{3} \widetilde{Q_{V}}-3.752 \times 10^{3}$.

\section{Conclusion}

In this study, we measured the streamwise velocity using PIV and obtained the shape index using the machine vision method. Subsequently, we computed the value of $\widetilde{Q_{V}}$ and $A P e$ using the continuous flow model and segregation model. And observed that

(1) The segregation pattern gradually evolves from core to streak, with the rotating speed decrease from a high value to a low. And the greater the rotating speed decrease, the more the number of streaks.

(2) Moreover, both the value and the fluctuation amplitude of the average streamwise velocity increase with the decrease of rotating speed at the center of the flow layer.

(3) After analyzing the relationship between $\widetilde{Q_{V}}$ and $\Lambda P e$ under different segregation patterns, we determined that the change in the rotating speed of the cylinder affects the interaction between diffusion and segregation in the flow layer. Furthermore, it affects the configuration of segregation patterns, which changes with $\widetilde{Q_{V}}$. The interaction between $P e$ and $\Lambda$ affects the amplitude of $\widetilde{Q_{V}}$, which can be comprehensively reflected by $\Lambda P e$. Additionally, $\widetilde{Q_{V}}$ is linearly proportional to $\Lambda P e$, i.e., $\Lambda P e=7.854 \times 10^{3} \widetilde{Q_{V}}-3.752 \times 10^{3}$.

We quantitated the relationship between $A P e$ and $\widetilde{Q_{V}}$ and obtained an expression for this relationship. This relationship provides an important basis theory for predicting the segregation pattern and improving the efficiency of industrial production. Granular segregation is not only 
related to granular velocity but also related to the granular temperature and concentration, the traditional granular temperature measurement technology obtained by PIV has a low spatial resolution, while speckle visibility spectroscopy (SVS) technology with high spatial resolution can be used to analyze the granular segregation dynamics.

\section{Acknowledgement}

This work has been supported by the National Natural Science Foundation of China (91634202, 11972212), the Science and Technology Commission of Shanghai Municipality (19142201500).

\section{Reference}

[1] J.M.N.T. Gray, Particle segregation in dense granular flows, Annual Review of Fluid Mechanics. 50 (1)

(2018) 122316-045201.

[2] S.H. Chou, F.C. Yang, S.S. Hsiau, Influence of interstitial fluid viscosity and particle size on creeping granular flow in a rotating drum, International Journal of Multiphase Flow. 113 (2019) 179190.

[3] D.V. Khakhar, A.V. Orpe, J.M. Ottino, Continuum model of mixing and size segregation in a rotating cylinder: Concentration-flow coupling and streak formation, Powder Technology. 116 (2-3) (2001) 232-245.

[4] A.G. Smart Towards a Microrheological Description of Granular Segregation. Aiche Meeting. 2009.

[5] K. Shinohara, K. Shoji, T. Tanaka, Mechanism of Size Segregation of Particles in Filling a Hopper, Industrial \& Engineering Chemistry Process Design and Development. 11 (3) (1972) 369-376.

[6] Y. Fan, K.M. Hill, Theory for shear-induced segregation of dense granular mixtures, New Journal of Physics. 13 (9) (2011) 095009. 
[7] M.J. Woodhouse, A.R. Thornton, C.G. Johnson, Segregation-induced fingering instabilities in granular freesurface flows, Journal of Fluid Mechanics. 709 (2012) 543-580.

[8] D. Tunuguntla, O. Bokhove, A. Thornton. D. R. Tunuguntla, O. Bokhove, Anthony Richard Thornton. A mixture theory for size and density segregation in shallow granular free-surface flows, Journal of Fluid Mechanics. 749 (2014) 99-112.

[9] A.R. Thornton, J.M.N.T. Gray, Breaking size segregation waves and particle recirculation in granular avalanches, Journal of Fluid Mechanics. 596 (596) (2008) 261-284.

[10] L. Prigozhin, H. Kalman, Radial mixing and segregation of a binary mixture in a rotating drum: Model and experiment, Physical Review E. 57 (2) (1998) 2073-2080.

[11] S. Chakraborty, P.R. Nott, J.R. Prakash, Analysis of radial segregation of granular mixtures in a rotating drum, The European Physical Journal E-Soft Matter. 1 (4) (2000) 265-273.

[12] G. Metcalfe, T. Shinbrot, J.J. Mccarthy, Avalanche mixing of granular solids, Nature. 374 (6517) (1995) 3941.

[13] J.M. Ottino, D.V. Khakhar, Mixing and segregation of granular materials, Annu.rev.fluid Mech. 32 (1) (2000) 55-91.

[14] J.M.N.T. Gray, P. Gajjar, P.B. Kokelaar, Particle-size segregation in dense granular avalanches. Comptes Rendus Physique. 16 (1) (2015) 73-85.

[15] J.M.N.T. Gray, A. R. Thornton, A theory for particle size segregation in shallow granular free-surface flows. Proceedings of the Royal Society A-Mathematical Physical and Engineering Sciences. 461 (2057) (2005) $1447-1473$. 
[16] L. Jing, C. Y. Kwok, Y. F. Leung, Micromechanical origin of grain size segregation. Physical Review Letters. 118 (2017) 118001.

[17] J.T. Jenkins, F. Mancini, Balance laws and constitutive relations for plane flows of a dense, binary mixture of smooth, nearly elastic, circular disks. Journal of Applied Mechanics. 54 (1) (1987) 27-34.

[18] D.V. Khakhar, J.J. Mccarthy, T. Shinbrot, et al, Transverse flow and mixing of granular materials in a rotating cylinder, Physics of Fluids. 9 (1) (1997) 31

[19] J.J. McCarthy, D.V. Khakhar, J.M. Ottino, Computational studies of granular mixing, Powder Technology. 109 (2000) 72-82.

[20] D.V. Khakhar, J.J. Mccarthy, J.F. Gilchrist, Chaotic mixing of granular materials in two-dimensional tumbling mixers, Chaos An Interdisciplinary Journal of Nonlinear Science. 9 (1) (1999)195.

[21] N. Jain, J.M. Ottino, R.M. Lueptow, Regimes of segregation and mixing in combined size and density granular systems: an experimental study, Granular Matter. 7 (2/3) (2005) 69-81.

[22] K.M. Hill, D.V. Khakhar, J.F. Gilchrist, Segregation-driven organization in chaotic granular flows, Proceedings of the National Academy of Sciences. 96 (21) (1999) 11701-11706.

[23] C.P. Schlick, Y. Fan, A.B. Isner, Modeling segregation of bidisperse granular materials using physical control parameters in the quasi-2D bounded heap, AIChE Journal. 61 (15) (2014) 1524-1534.

[24] C.P. Schlick, Y. Fan, P.B. Umbanhowar, Granular segregation in circular drums: theoretical model and scaling laws, Journal of Fluid Mechanics. 765 (2015) 632-652.

[25] I. Zuriguel, J.M.N.T. Gray, J. Peixinho, Pattern selection by a granular wave in a rotating drum, Physical Review E. 73 (6) (2006) 061302. 
[26] K.M. Hill, G. Gioia, D. Amaravadi, et al, Moon patterns, sun patterns, and wave breaking in rotating granular mixtures, Complexity. 10 (4) (2010) 79-86.

[27] N.A. Pohlman, S.W. Meier, R.M. Lueptow, Surface velocity in three-dimensional granular drums, Journal of Fluid Mechanics. 560 (2006) 355-368.

[28] D.V. Khakhar, J.J. Mccarthy, J.M. Ottino, Radial segregation of granular mixtures in rotating cylinders, Physics of Fluids. 9 (12) (1997) 3600.

[29] N. Jain, J.M. Ottino, R.M. Lueptow, Regimes of segregation and mixing in combined size and density granular systems: an experimental study, Granular Matter. 7 (2/3) (2005) 69-81.

[30] C.C. Liao, S.S. Hsiau, H.C. Nien, Effects of density ratio, rotation speed, and fill level on density-induced granular streak segregation in a rotating drum, Powder Technology. 284 (2015) 514-520.

[31] C.C. Liao, S.S. Hsiau, H.C. Nien, Density-driven spontaneous streak segregation patterns in a thin rotating drum, Physical Review E. 89(2014) 062204.

[32] W. Thielicke, E.J. Stamhuis, PIVlab - Towards user-friendly, affordable and accurate digital particle image velocimetry in MAT-LAB, open research software. 2 (1) (2014) 355-364.

[33] Z. B. Chen, K. G. Li, M. Omidvar, et al, Guidelines for DIC in geotechnical engineering research, International Journal of Physical Modelling in Geotechnics. (2016) 1-20.

[34] G. Devis, B. Wernher, T. B. Elisabeth, et al, Performance of PIV and PTV for granular flow measurements, Granular Matter. 19 (3) (2017) 42.

[35] Y. Fan, C.P. Schlick, P.B. Umbanhowar, et al. Modelling size segregation of granular materials: the roles of segregation, advection and diffusion. Journal of Fluid Mechanics, 741 (2014) 252-279. 
- Segregation patterns were quantified by the shape index.

- Averaged volume flow rate is proportional to the rotation speed.

- The Ratio of segregation to diffusion effects increased with the rotating speed.

- Averaged volume flow rate was linear with ratio of segregation to diffusion effects. 


\section{Figures}

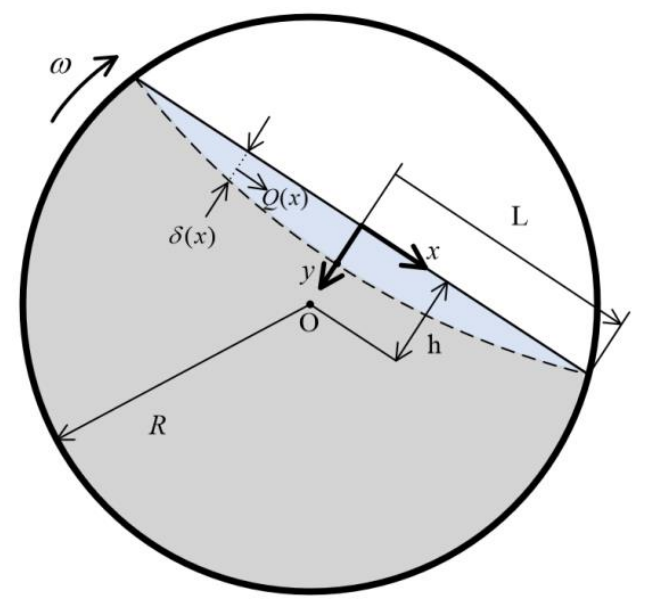

Fig. 1 Schematic representation of the rotating drum.

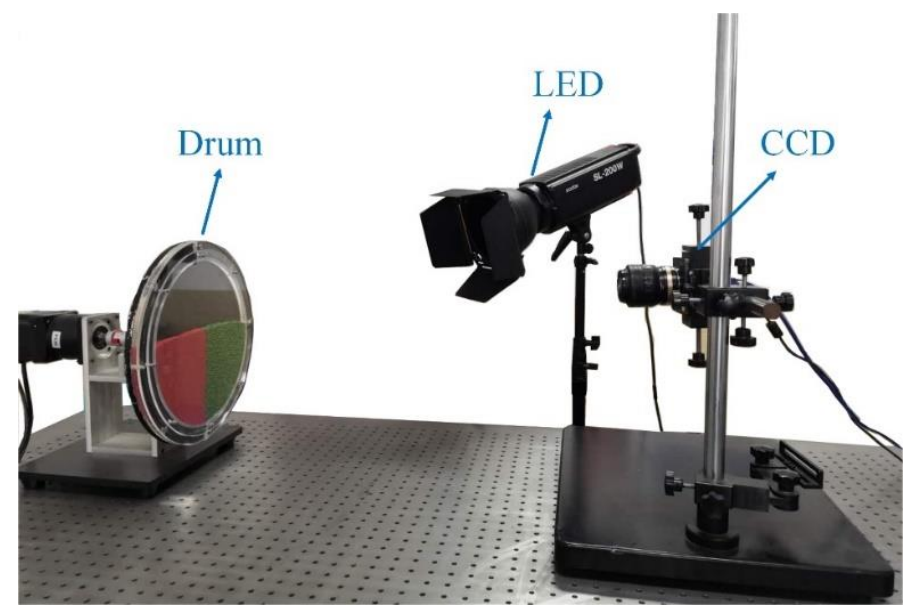

Fig. 2 Schematic diagram of the rotating drum and imaging system.

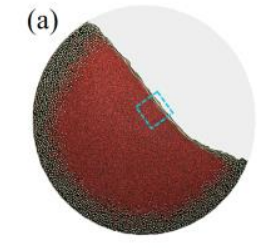

$\omega=1.02 \mathrm{rad} / \mathrm{s}$

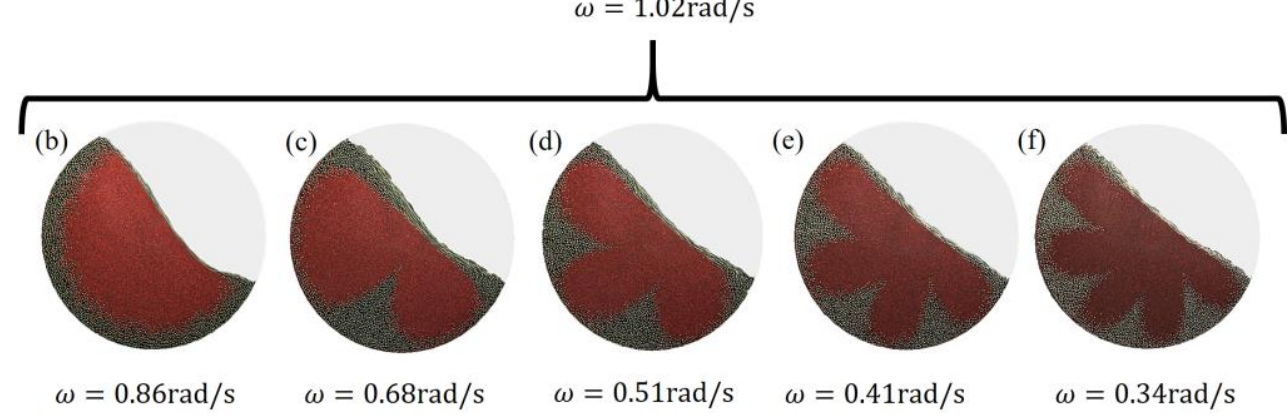

Fig. 3 Segregation patterns under different rotating speeds. Rotating speed of (a) $1.02 \mathrm{rad} / \mathrm{s}$; (b) $0.86 \mathrm{rad} / \mathrm{s}$; (c) $0.68 \mathrm{rad} / \mathrm{s}$; (d) $0.51 \mathrm{rad} / \mathrm{s}$; (e) $0.41 \mathrm{rad} / \mathrm{s}$; and (f) $0.34 \mathrm{rad} / \mathrm{s}$. (a) as the base pattern, (b) -(f) as the developed pattern after modulating the rotation speed, and the area of the blue dotted line is the measurement field. 
(a)

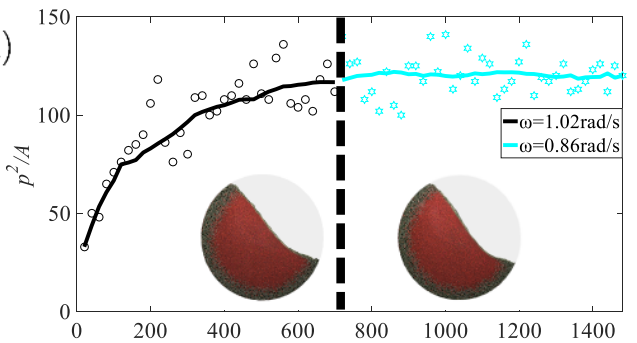

(b)

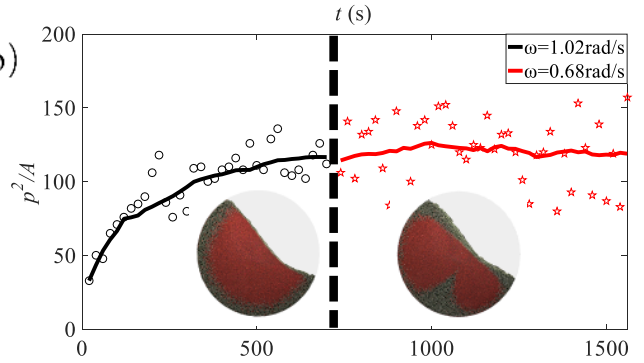

(c)

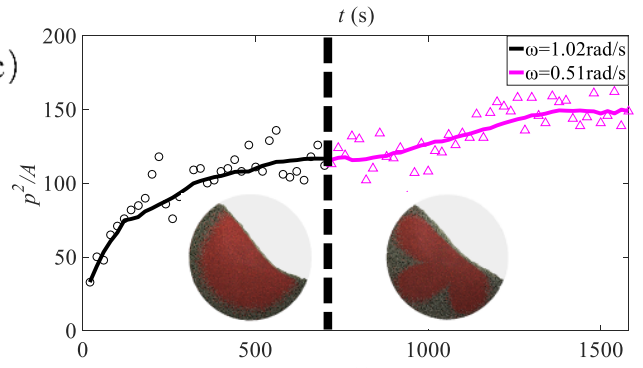

(d)

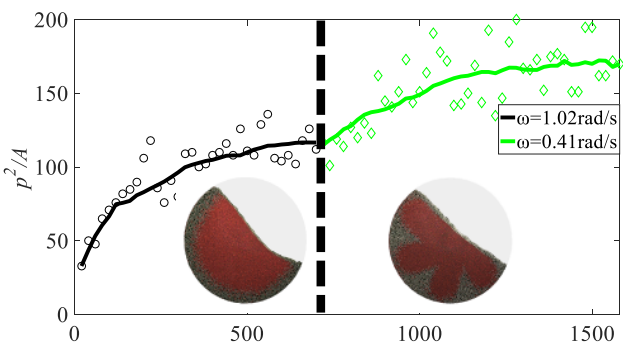

(e)

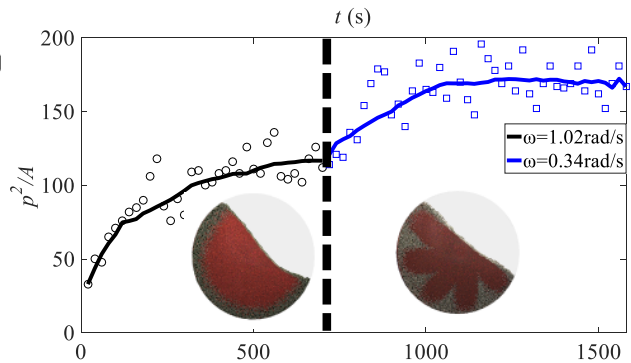

$t(\mathrm{~s})$

$t(\mathrm{~s})$

Fig. 4 Shape index under different rotating speeds. Modulation speed of (a) $0.86 \mathrm{rad} / \mathrm{s}$; (b) $0.68 \mathrm{rad} / \mathrm{s}$; (c) 0.51 $\mathrm{rad} / \mathrm{s}$; (d) $0.41 \mathrm{rad} / \mathrm{s}$; and (e) $0.34 \mathrm{rad} / \mathrm{s}$.

(a)

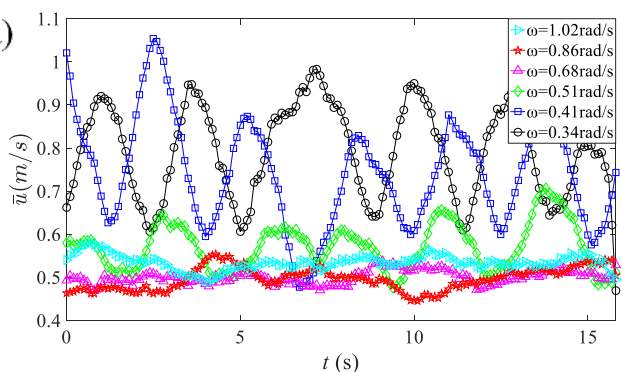

(b)

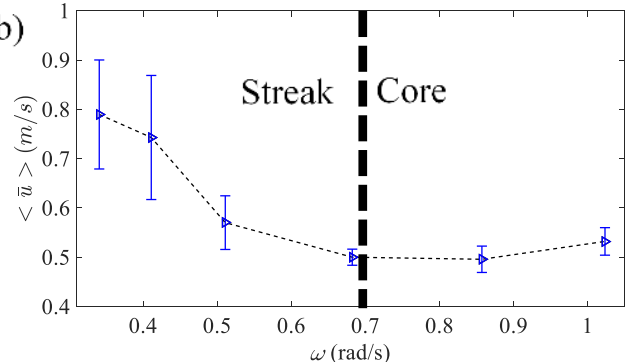

(c)

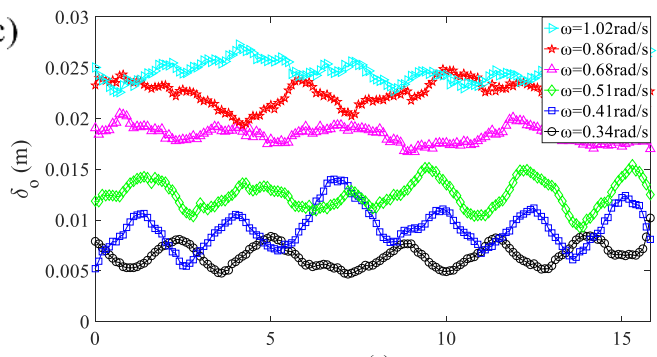

(d)

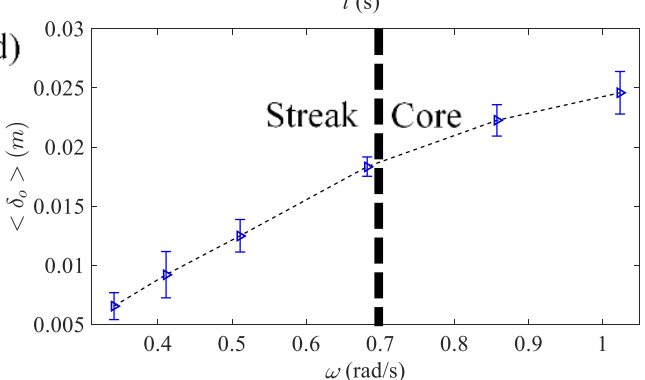

Fig. 5 Average streamwise velocity $\bar{u}$ distribution under different segregation patterns with respect to different rotation speeds (a). Time averaged $\bar{u}$ under different rotation speeds (b). The maximum thickness of the flow layer $\delta_{0}$ under different rotation speeds (c). Time averaged $\delta_{0}$ under different rotation speeds (d). 


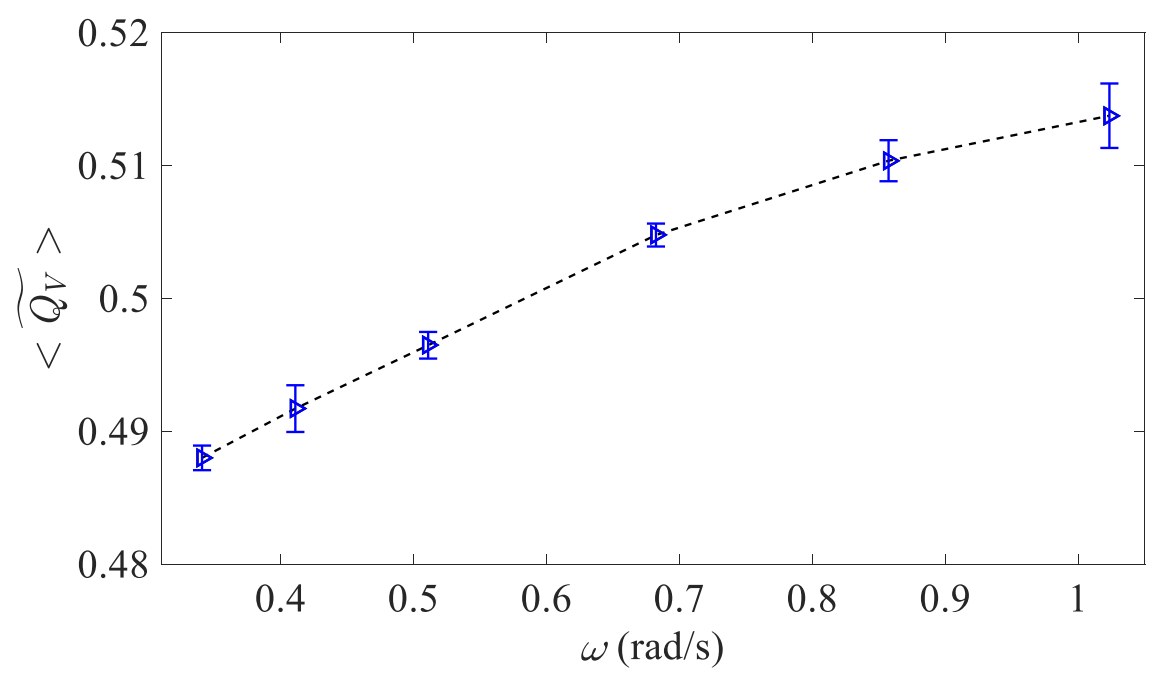

Fig. 6 Averaged volume flow rate $\left\langle\widetilde{Q_{V}}>\right.$ for different rotation speeds.
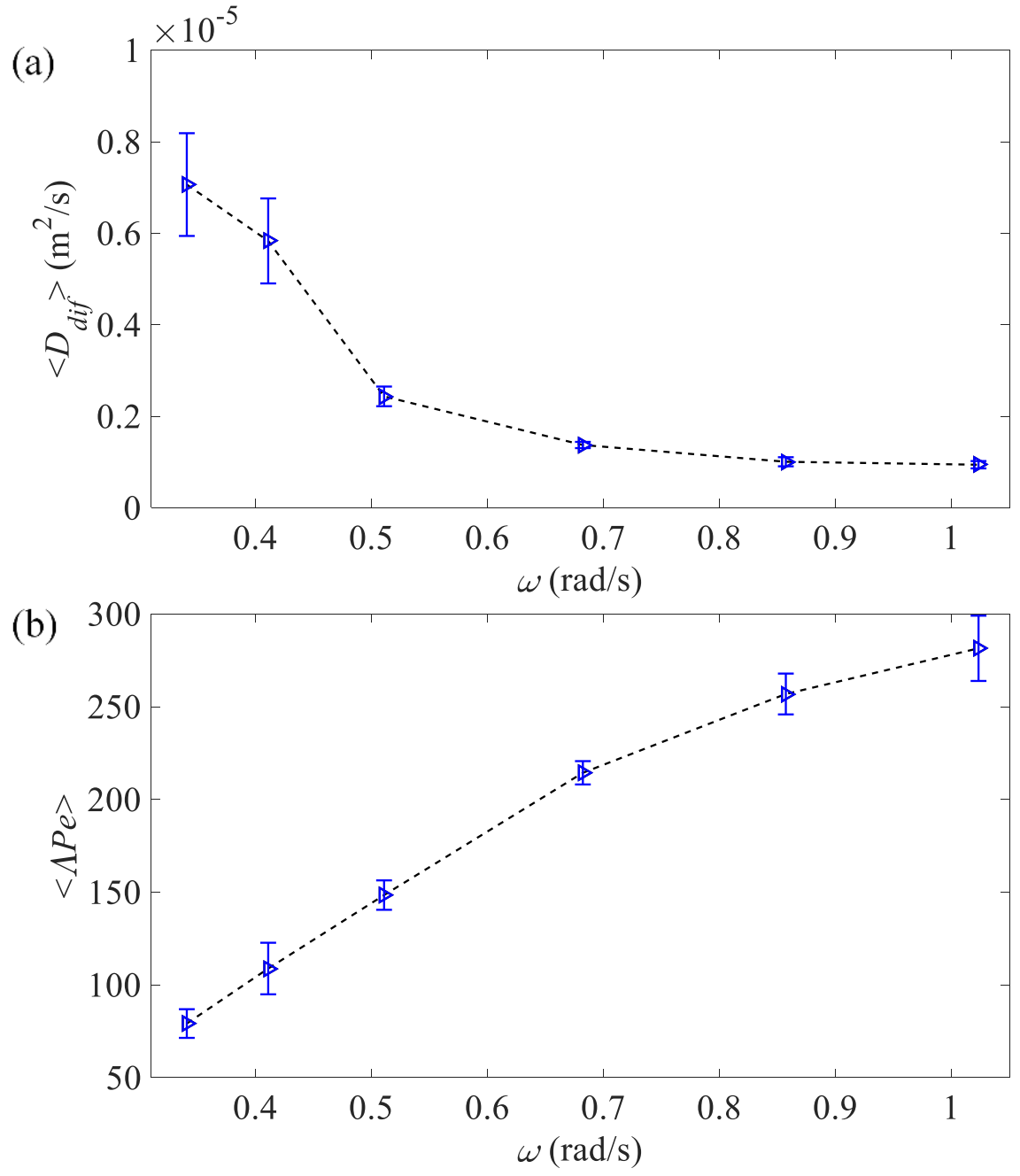

Fig. 7 Averaged diffusion coefficient $\left\langle D_{\text {dif }}\right\rangle$ for different rotation speeds (a); Averaged the ratio of segregation effects to diffusion effects $\langle\Lambda \mathrm{Pe}\rangle$ for different rotation speeds (b). 


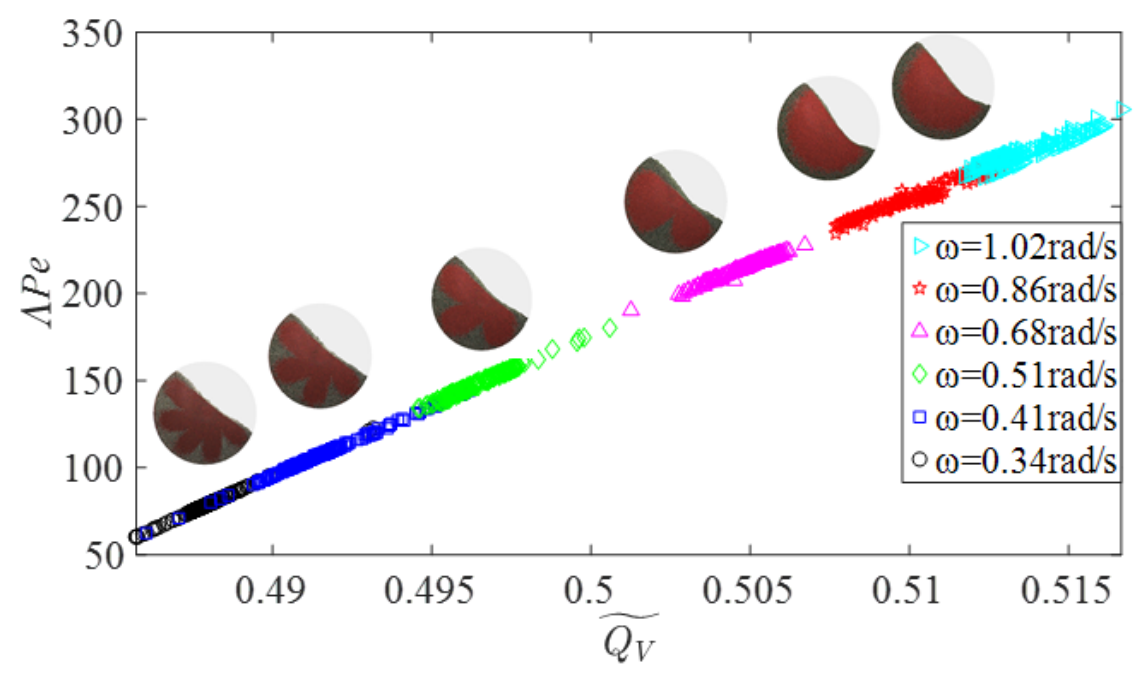

Fig. $8 \Lambda P e$ vs $\widetilde{Q_{V}}$ under different segregation patterns with respect to different rotational speeds. 